\title{
Multiplexed Anticounterfeiting Meta-image Displays with Single-sized Nanostructures
}

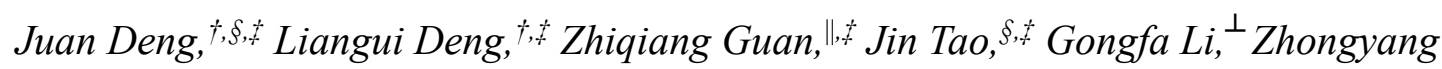

$\mathrm{Li}^{\dagger}{ }^{\dagger}$ Zile Li, ${ }^{, * *}$ Shaohua Yu, ${ }^{,, *}$ and Guoxing Zheng ${ }^{\dagger, \xi, *}$

${ }^{\dagger} E$ lectronic Information School, Wuhan University, Wuhan 430072, China

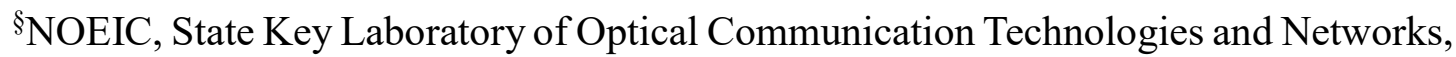
Wuhan Research Institute of Posts \& Telecommunications, Wuhan 430074, China

"School of Physics and Technology, Center for Nanoscience and Nanotechnology, and Key Laboratory of Artificial Micro- and Nano-structures of Ministry of Education, Wuhan University, Wuhan 430072, China

${ }^{\perp}$ Key Laboratory of Metallurgical Equipment and Control Technology of Ministry of Education, Wuhan University of Science and Technology, Wuhan 430081, China

J.D., L.G.D., Z.Q.G., and J.T. contributed equally

1. A general concept of the dual-channel anticounterfeiting metasurface by polarization control

The Jones matrix of an anisotropic nanostructure with an in-plane orientation $\theta$ can be expressed as

$$
T(\theta)=R(-\theta) T_{0} R(\theta)=\left[\begin{array}{cc}
\cos \theta & -\sin \theta \\
\sin \theta & \cos \theta
\end{array}\right] \cdot\left[\begin{array}{cc}
A & 0 \\
0 & B
\end{array}\right] \cdot\left[\begin{array}{cc}
\cos \theta & \sin \theta \\
-\sin \theta & \cos \theta
\end{array}\right],
$$

where $R(\theta)$ is the rotation matrix, $A$ and $B$ are the complex transmission (or reflection) coefficients of the nanostructure along with the long and short axes, respectively.

If the incident light passes through a polarizer, an anisotropic nanostructure and a bulky analyzer sequentially, the Jones vector of output light can be expressed as 


$$
J=\left[\begin{array}{cc}
\cos ^{2} \alpha_{2} & \sin \alpha_{2} \cos \alpha_{2} \\
\sin \alpha_{2} \cos \alpha_{2} & \sin ^{2} \alpha_{2}
\end{array}\right] \cdot T(\theta) \cdot\left[\begin{array}{c}
\cos \alpha_{1} \\
\sin \alpha_{1}
\end{array}\right],
$$

where $\alpha_{1}$ and $\alpha_{2}$ represent the directions of transmission axis of the polarizer and analyzer, respectively. If the light intensity after the polarizer is $I_{0}$, we can deduce the expression of output light intensity according to Eq. S2 as

$$
I=I_{0}\left[\frac{A-B}{2} \cos \left(2 \theta-\alpha_{2}-\alpha_{1}\right)+\frac{A+B}{2} \cos \left(\alpha_{2}-\alpha_{1}\right)\right]^{2} .
$$

We find that any anisotropic nanostructure $(A \neq B)$ can be used for a continuous intensity modulation when the light intensity $I_{0}$, the transmission axes of the polarizer and analyzer are unambiguously given.

\section{1 dual-channel anticounterfeiting metasurfaces}

When the transmission axis of the polarizer is perpendicular to the transmission axis of the analyzer, we can simplify Eq. S3 as

$$
I_{1}=I_{0}\left(\frac{A-B}{2}\right)^{2} \cos ^{2}\left(2 \theta-\alpha_{2}-\alpha_{1}\right) .
$$

Next, if we rotate the nanobrick by an angle such as $\pi / 8$ around the optical axis, the corresponding intensity of output light is changed to

$$
I_{2}=I_{0}\left(\frac{A-B}{2}\right)^{2} \cos ^{2}\left(2 \theta-\alpha_{2}-\alpha_{1}-\frac{\pi}{4}\right) .
$$

Specially, if the nanobrick acts as an ideal half-wave plate (i.e., $A=1$ and $B=-1$ ) and $\alpha_{2}=-\alpha_{1}=\frac{\pi}{4}$, we can simplify Eq. S4 and Eq. S5 as

$$
\begin{aligned}
& I_{1}=I_{0} \cos ^{2}(2 \theta) \text { and } \\
& I_{2}=I_{0} \cos ^{2}\left(2 \theta-\frac{\pi}{4}\right) .
\end{aligned}
$$

From Eqs. S6-7 we can find that there are four orientation candidates in its defined interval $[0, \pi]$ to generate an equal output intensity. The orientation degeneracy provides a new degree of freedom, which will benefit for encoding a continuous grayscale image into channel 1 and an independent two-step image into channel 2 with a single piece of metasurface. More details about the working principle have been demonstrated in the main text.

\section{2 dual-channel anticounterfeiting metasurfaces with watermarks}

If the nanobrick acts as an ideal polarizer (i.e., $A=1$ and $B=0$ ) and $\alpha_{1}=\alpha_{2}+\frac{\pi}{2}=\frac{\pi}{2}$, we can simplify Eq. S3 as

$$
I_{3}=I_{0} \sin ^{2} 2 \theta .
$$

If the transmission axis of the analyzer is rotated from zero degree to $\frac{\pi}{4}$, Eq. S3 can be expressed as

$$
I_{4}=I_{0}\left[\cos \left(2 \theta-\frac{3 \pi}{4}\right)+\frac{\sqrt{2}}{2}\right]^{2} \text {. }
$$

Based on three kinds of relationships (equal, greater and less) between $I_{3}$ and $I_{4}$ at the 
orientation interval $[0, \pi]$, shown in Fig. $4 \mathrm{~b}$ of the main text, the channel switching between a continuous grayscale image and a continuous grayscale image with watermark information can be achieved.

Table S1 | Summary of the dual-channel anticounterfeiting metasurface design.

\begin{tabular}{|c|c|c|c|}
\hline Scheme & Nanobrick & Channel & Intensity of output light \\
\hline $\begin{array}{l}\text { Metas } \\
\text { Polari }\end{array}$ & & 1 & $I_{1}=I_{0} \cos ^{2}(2 \theta)$ \\
\hline & $\begin{array}{l}\text { Half- } \\
\text { waveplate }\end{array}$ & 2 & $I_{2}=I_{0} \cos ^{2}\left(2 \theta-\frac{\pi}{4}\right)$ \\
\hline & & 1 & $I_{3}=I_{0} \sin ^{2} 2 \theta$ \\
\hline & Polarizer & 2 & $I_{4}=I_{0}\left[\cos \left(2 \theta-\frac{3 \pi}{4}\right)+\frac{\sqrt{2}}{2}\right]$ \\
\hline
\end{tabular}

\section{Flowchart for anticounterfeiting metasurface design}

\subsection{Metasurface design for sample 1}

For sample 1, we encode a continuously grayscale image (a lotus picture) into channel 1 and an anticounterfeiting fringe pattern into channel 2. Firstly, according to the target grayscale image $\left(I_{1}\right)$ and the intensity modulation equation $I_{1}=I_{0} \cos ^{2}(2 \theta)$, we can calculate the initial distribution of orientations $\theta$, in which all orientations lie in the interval $[0, \pi / 4]$. Then, the binary anticounterfeiting fringes $\left(I_{2}\right)$, which is designed by professional anticounterfeiting fringes design software, is added as a criterion to determine the new orientation distribution $\theta_{\text {new. }}$. Specifically, if the intensity value of $I_{2}$ is 0 (a "lower" value), the corresponding initial orientation $\theta$ is changed to be $\pi / 2-\theta$ or $\pi-\theta$, in which we can randomly choose one of two orientation candidates. If the intensity value of $I_{2}$ is 255 (a "higher" value), the corresponding initial orientation remains unchanged or is changed to $\pi / 2+\theta$, which can be chosen randomly. Thus, we get the final orientation distribution $\theta_{\mathrm{f}}$. After completing above operation cell-by-cell, we can get all orientations of nanostructures for sample 1 . 


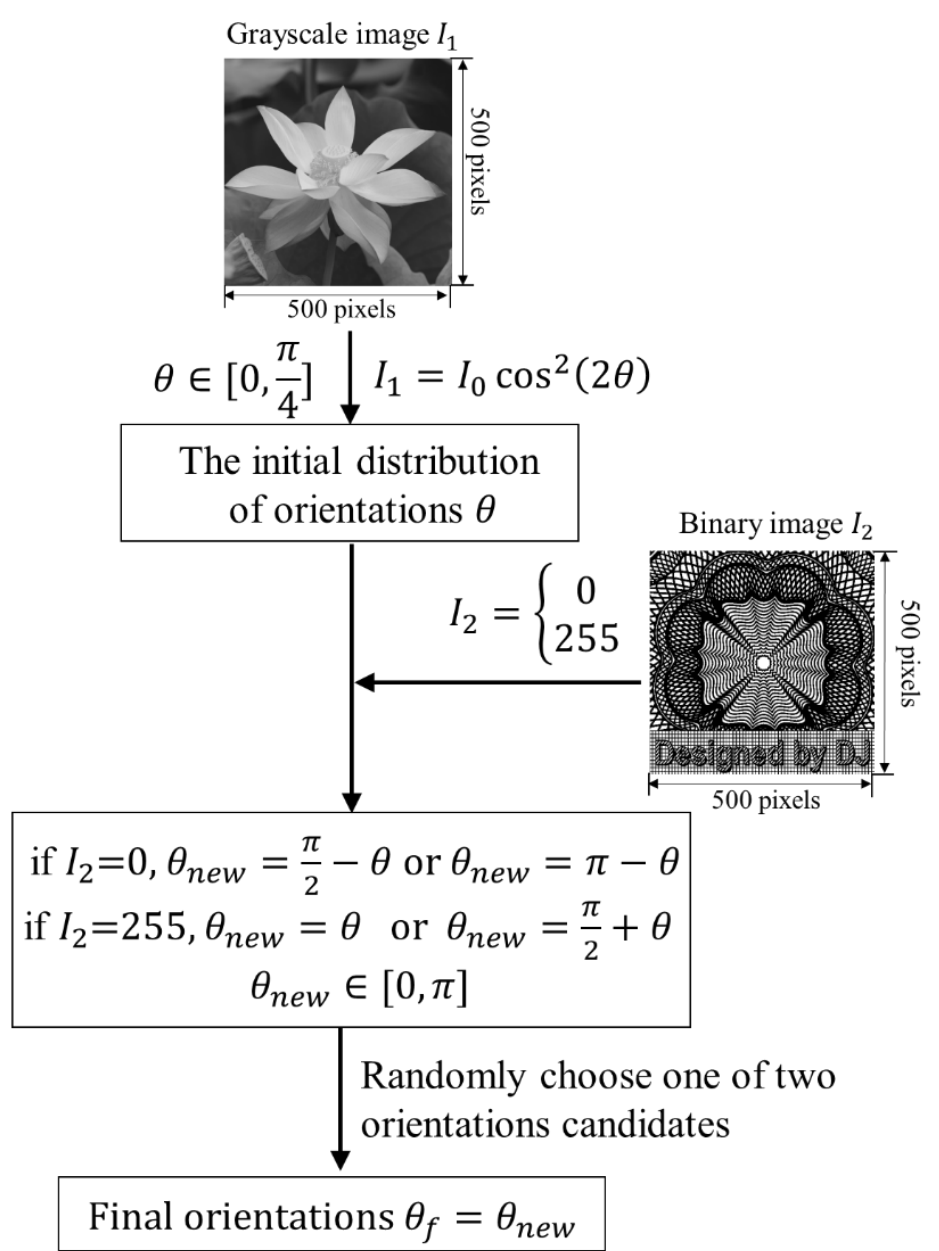

Figure S1. Flowchart for sample 1 design.

\subsection{Metasurface design for sample 2}

Unlike sample 1, the anticounterfeiting pattern of sample 2 is partially embedded into a continuous grayscale image (a lotus picture) as a watermark, which makes parts of the lotus image in channel 2 still be recognized. The flowchart of designing sample 2 is shown in Fig. S2. Firstly, we calculate the initial distribution of orientations $\theta$ according to the grayscale image $\left(I_{1}\right)$ and the intensity modulation equation $I_{1}=I_{0} \cos ^{2}(2 \theta)$, in which all orientations lie in the interval $[0, \pi / 4]$. Then, if the pixel is located inside the red rectangle, the initial orientation $\theta$ is converted to $\theta_{\text {new }}$ according to a criterion. Specifically, if the inensity value of $I_{2}$ is 0 , the corresponding initial orientation is changed to be $\pi / 2-\theta$ or $\pi-\theta$, in which we can randomly choose one of the two candidates. If the intensity value of $I_{2}$ is 255 , the corresponding initial orientation remains unchanged or is changed to be $\pi / 2+\theta$, which can be chosen randomly. If a pixel is located outside the red rectangle, all corresponding initial orientation $\theta$ remains unchanged. Based on the above steps, we can obtain the finally orientation distribution $\theta_{\mathrm{f}}$, which satisfies the requirement of encoding a continuous grayscale image into channel 1 and a traditional Chinese paper-cut pattern embedded into the continuous grayscale image in channel 2. After finishing above operation cell-by-cell, we can get all orientations of nanostructures for sample 2 . 


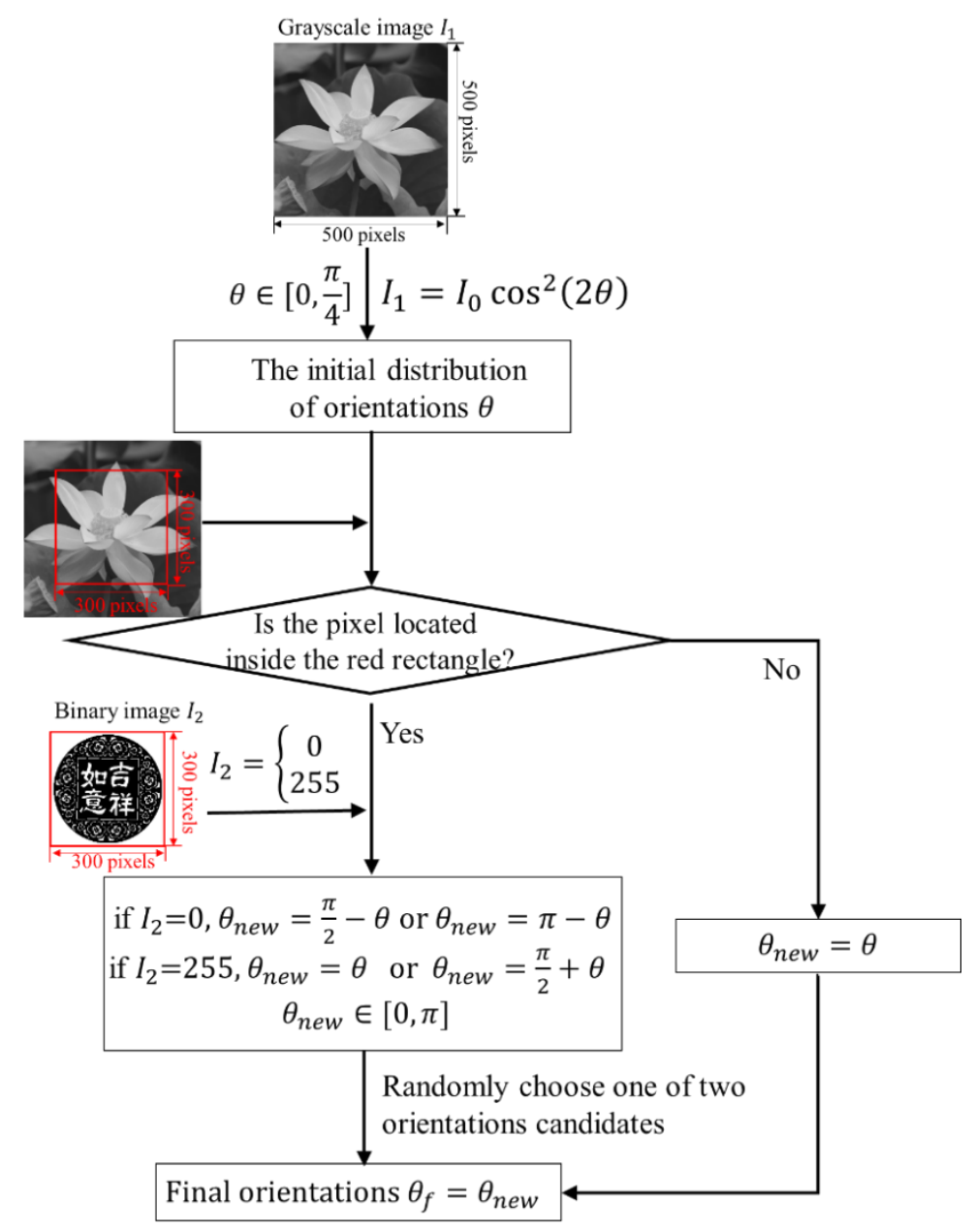

Figure S2. Flowchart for sample 2 design.

\subsection{Metasurface design for sample 3}

In sample 3, there are more elements can be randomly designed. To generate a two-step anticounterfeiting pattern, we firstly need to produce a series of circles with different radiuses and center positions, which will benefit for dividing the whole region into various irregular blocks. Then, we randomly binarize these irregular patches to obtain $I_{2}$. In addition, we can also merge the image $I_{3}$ into the image $I_{2}$ to obtain the desired binary anticounterfeiting pattern $I_{4}$, which is used as a criterion to determine the new orientation distribution $\theta_{\text {new }}$. Next, based on the same design method of sample 1 mentioned above, we can obtain the final distribution of orientation $\theta_{\mathrm{f}}$, which meets the requirement of simultaneously encoding a continuous grayscale image and a two-step anticounterfeiting pattern consisting of various irregular geometry patterns into a single piece of metasurface. 


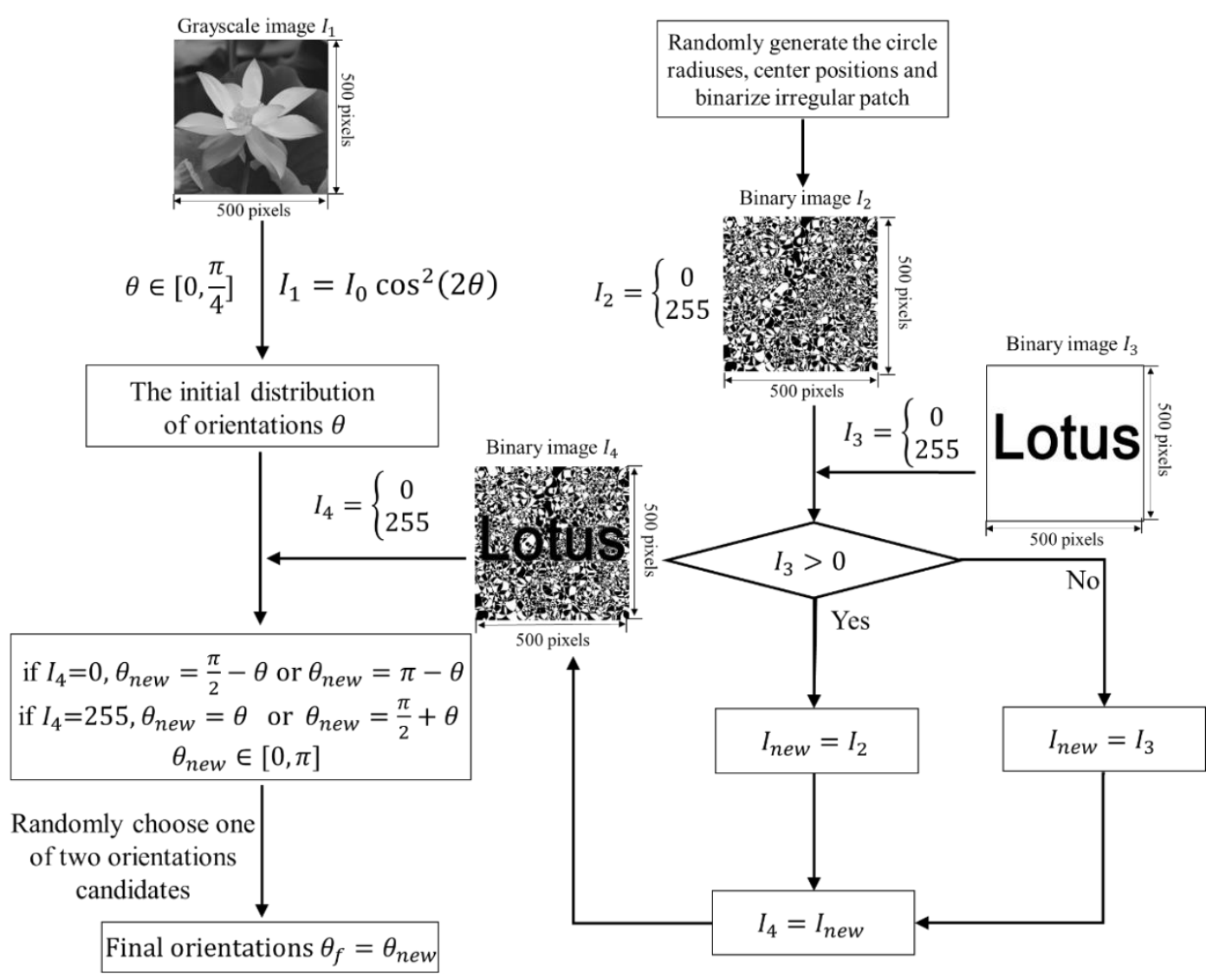

Figure S3. Flowchart for sample 3 design.

\section{Scanning electron microscopy image}

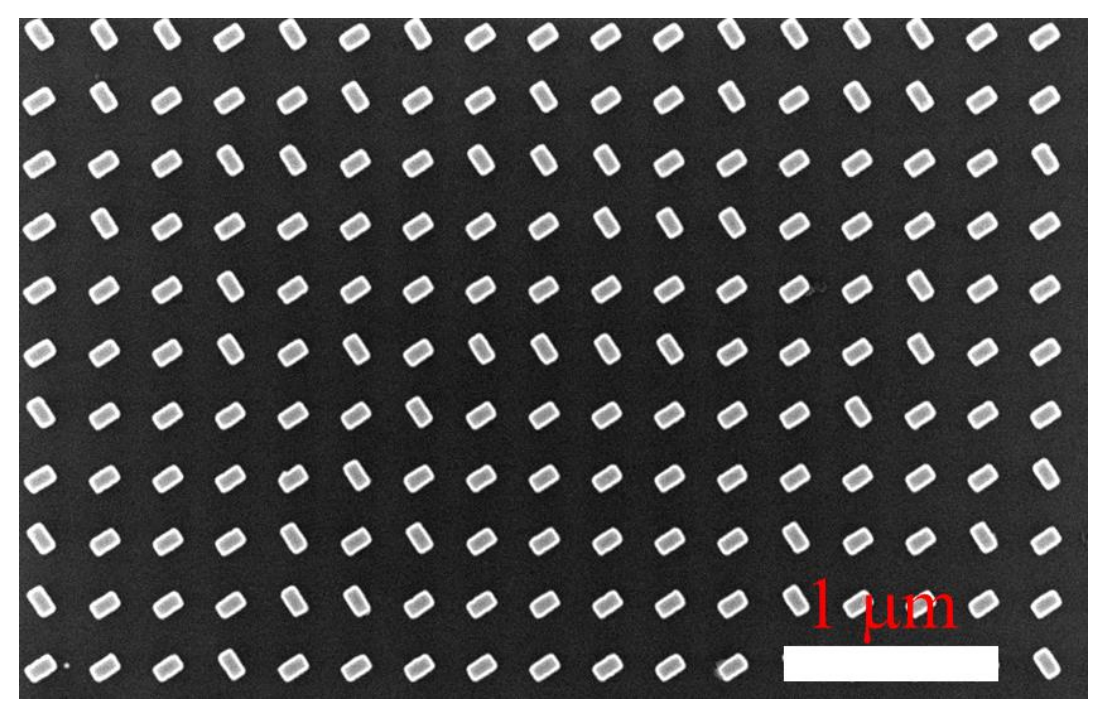

Figure S4. Scanning electron microscopy (SEM) image of sample 1 (partial view). The scale bar is $1 \mu \mathrm{m}$.

\section{Experimental setup for characterization of the anticounterfeiting metasurfaces}

To decode information hided in the anticounterfeiting metasurface, we use an optical microscope 
(Motic BA310MET-T) as shown in Fig. S5a to capture the meta-images. The optical layout is shown in Fig. S5b. A polarizer is placed after a red filter and an aperture to produce the desired linearly polarizer light. An analyzer is placed before the objective lens. The images are collected by an objective lens and recorded by a CMOS camera.

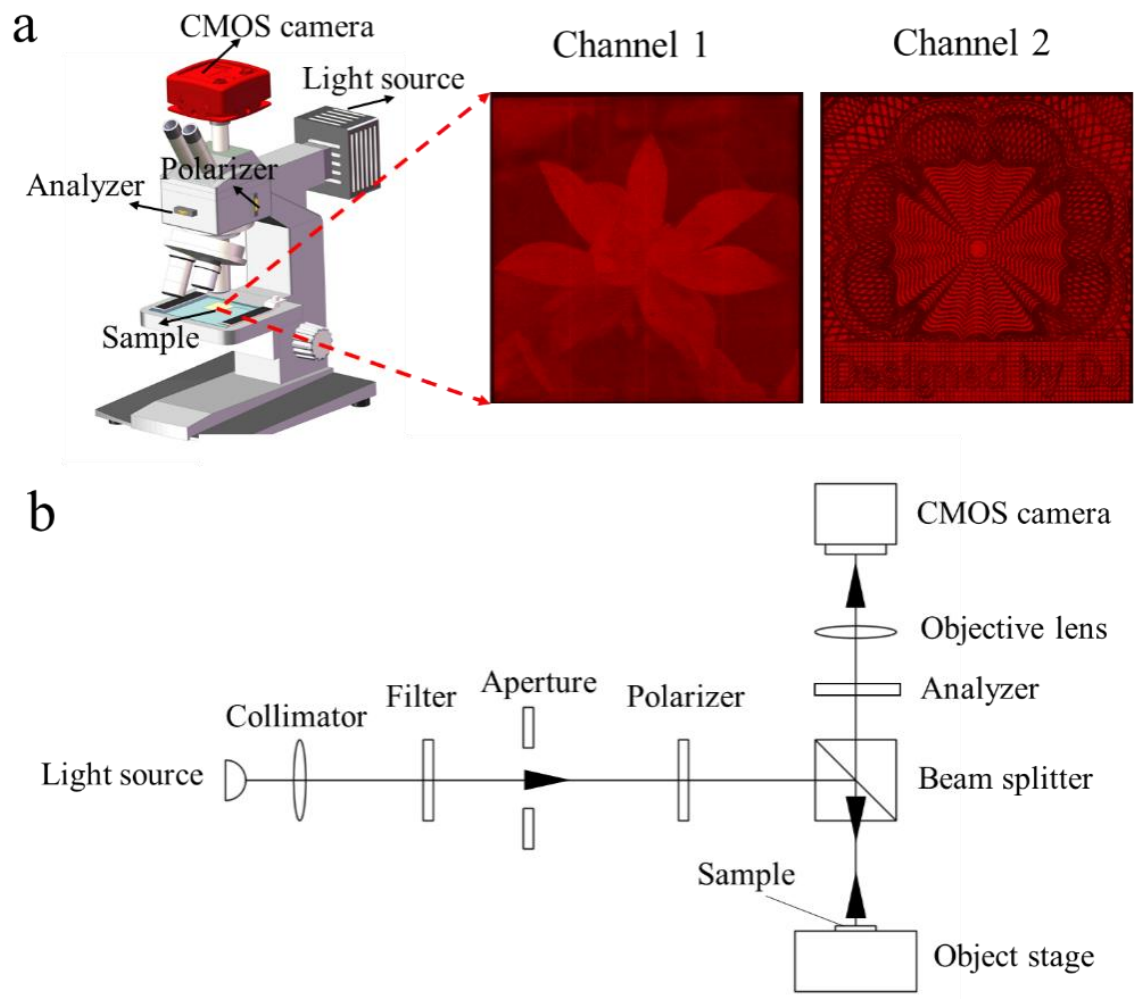

Figure S5. Experimental setup for characterization of the anticounterfeiting metasurface.

\section{Anticounterfeiting metasurfaces with various randomly configured background}

\section{patterns}

A notable feature of our proposed dual-channel anticounterfeiting metasurfaces is that the background patterns of channel 2 consist of some random elements. In addition to different sizes of circles, it can be designed with different sizes of squares or triangles, even random black-and-white points, as shown in Fig. S6. For design A-C, we encode the same grayscale image (a lotus picture) into channel 1 but different two-step images into channel 2. The background patterns of design A$\mathrm{C}$ are designed with different sizes of squares, triangles and randomly configured black-and-white points, respectively. Furthermore, the size and position of these elements of background pattern can be randomly chosen (design D-F), so that each image has a unique identifier to avoid being copied, which greatly increases the security of an anticounterfeiting pattern. 


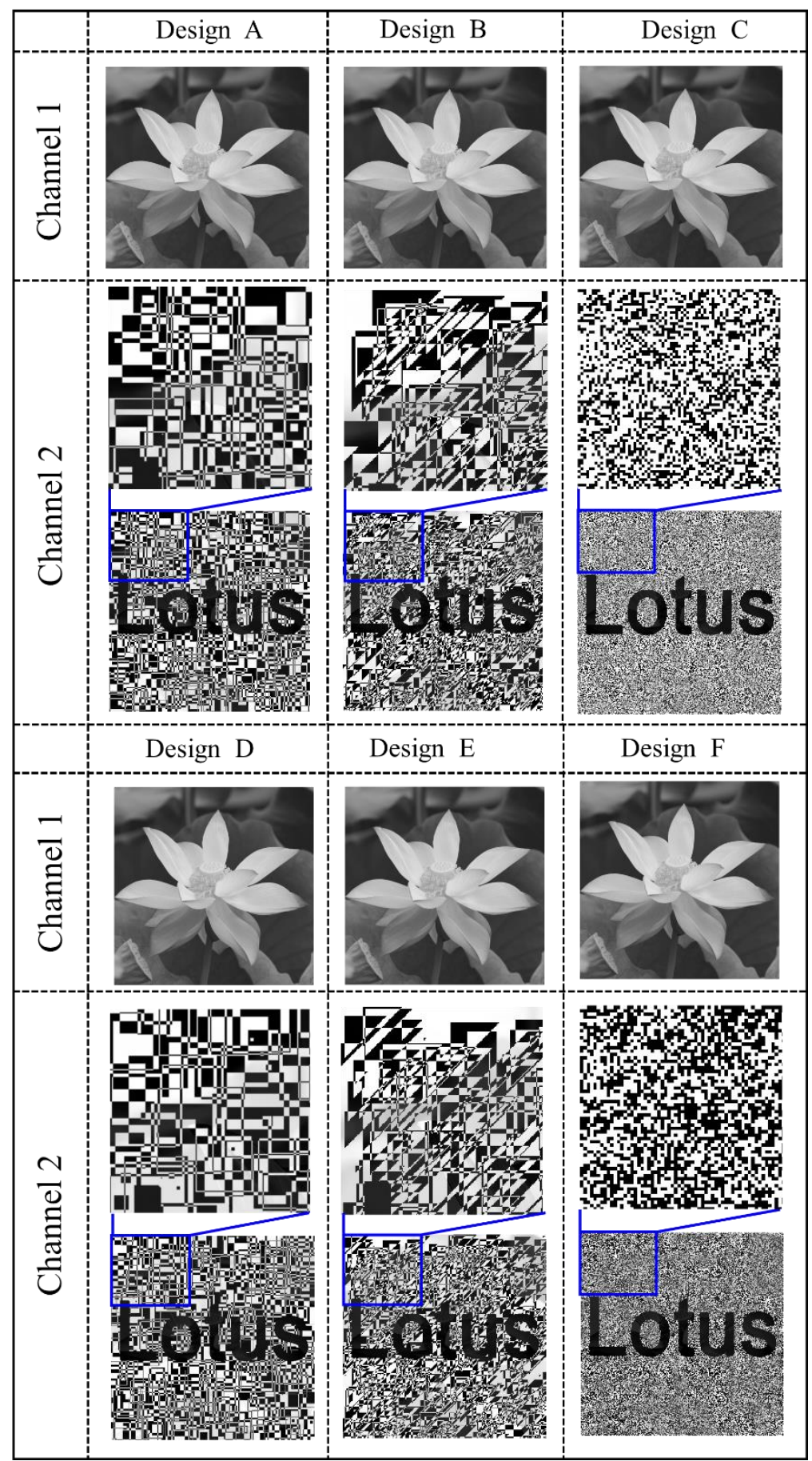

Figure S6. Simulation results for anticounterfeiting metasurfaces with various randomly configured background patterns. For design A-C, there is the same grayscale image (a lotus picture) in channel 1 but different two-step images in channel 2, whose background patterns are designed with different sizes of squares, triangles or random black-and-white points, respectively. To prove the randomness of the background elements, design C-F are generated as a control group, in which only the background patterns of channel 2 are designed to be different from the design A-C.

\section{Broadband response of the dual-channel anticounterfeiting metasurfaces}

To investigate the broadband response of the dual-channel anticounterfeiting metasurfaces, we 
acquire the meta-images under illumination by a quartz halogen lamp (light source of the experimental optical microscope). Figure. S7 shows that the meta-images of two channels are clearly observed.

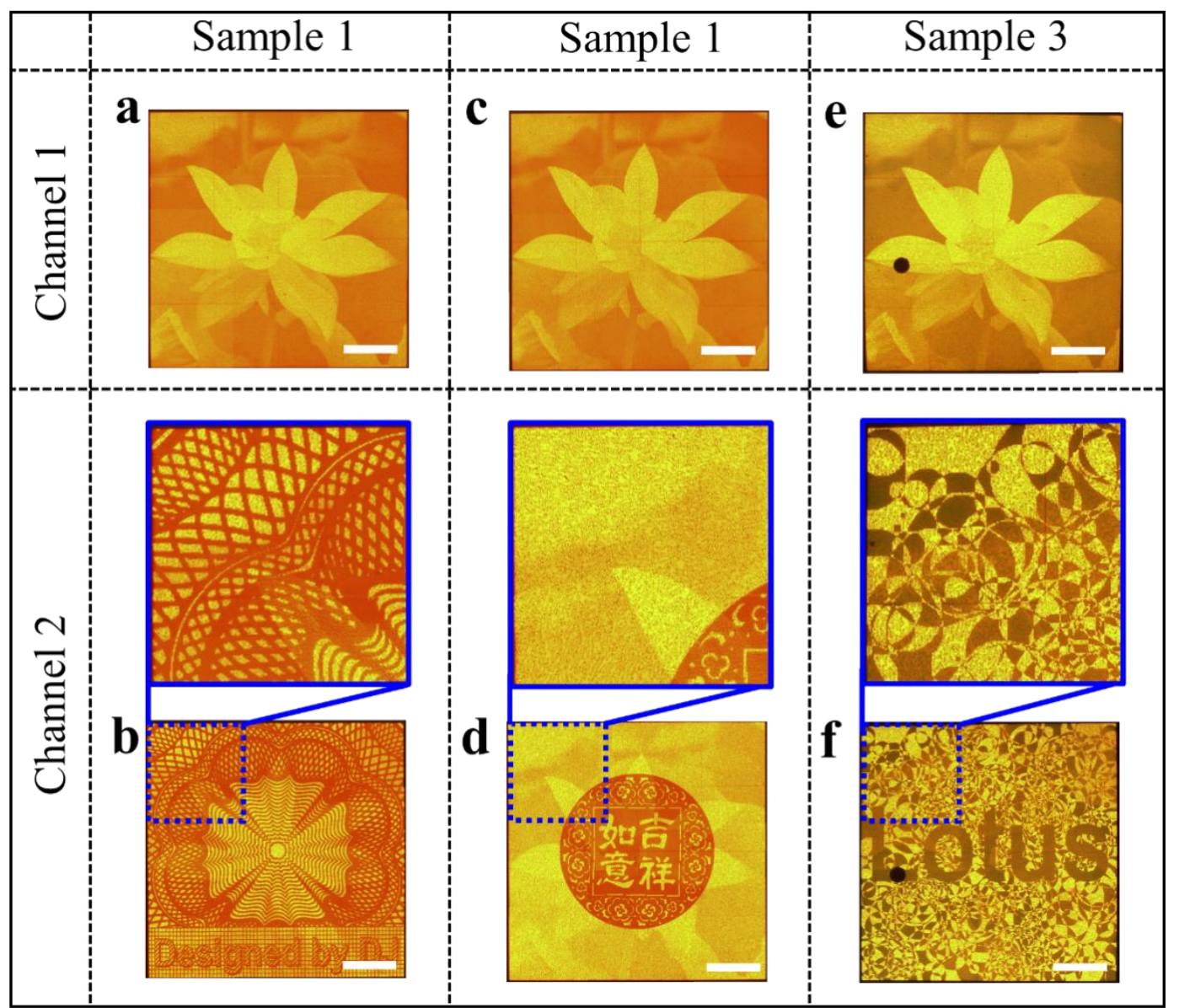

Figure S7. Experimental results of the dual-channel anticounterfeiting metasurface under illumination of a quartz halogen lamp. The first row shows the experimentally decoded meta-images when three samples are put into an orthogonal-polarization light path, in which the direction of transmission axis of the polarizer and the analyzer are $-\pi / 4$ and $\pi / 4$, respectively. The second and third rows present zoom-in and total views of the experimentally decoded meta-images by rotating the sample around the optical axis by $\pi / 8$, respectively. Three samples are designed and fabricated with dimensions of $200 \times 200 \mu \mathrm{m}^{2}$. The scale bar is $40 \mu \mathrm{m}$.

\section{A design scheme to solve the extremum-mapping problem}

According to Eqs. S6-7, we can see that the key point of our approach is establishing the one-tofour corresponding between output light intensity and nanobrick orientations. For extreme values such as 0 and 1, there are only two candidates rather than four. In addition, their corresponding "higher" and "lower" values in channel 2 are equal to 0.5 , which may partially blur the image corresponding to these pixels in channel 2. Therefore, for a continuous grayscale image with seldom extreme values of 0 or 1 , our scheme is still applicable and our experimental results have proved it.

If we have a continuous image with abundant grayscales of 0 and 1 , our proposed scheme shown in Fig. 4b in the main text is applicable. As shown in Fig. S8, there exist three zones (\#1, \#2 and \#3) to meet the requirement of encoding a continuous grayscale image into channel 1, while a two-step 
image in channel 2. Specially, for some extreme values of channel 1, there exist corresponding higher and lower values in channel 2 to generate a binary image, as shown in Fig. S8b.

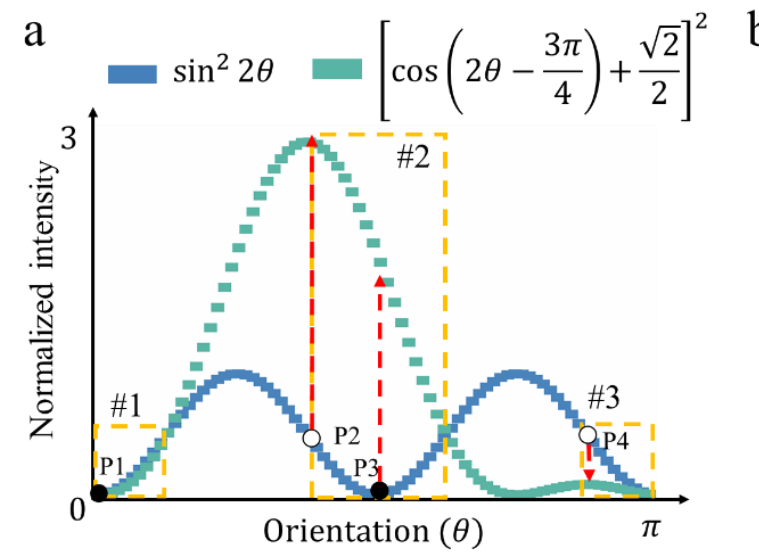

b
\begin{tabular}{|c|c|c|}
\hline & Channel 1 & Channel 2 \\
\hline P1 & 0 (lower value) & 0 (lower value) \\
\hline P2 & 0.5 (higher value) & 2.80 (higher value) \\
\hline P3 & 0 (lower value) & 2 (higher value) \\
\hline P4 & 0.5 (higher value) & 0.07 (lower value) \\
\hline
\end{tabular}

Figure S8. Principle of solving the extremum-mapping problem. P1 and P3 are the extreme value points with a value of "0" in channel 1, but correspond to lower and higher values in channel 2, respectively. P2 and P4 are the extreme value points with a value of " 1 " in channel 1, but correspond to higher and lower values of channel 2 , respectively.

\section{Experimental results of the dual-channel meta-image displays working in transmission}

mode

According to Eqs. 4 and 5, we can obtain the simulation results of sample four, as shown in Fig. S9 a-b. With the help of an optical microscope, the experimental results in transmission mode can be obtained, as shown in Fig. S9c-f. Obviously, when the transmission axes of a polarizer and an analyzer are mutually orthogonal, all grayscale images captured with and without a red light filter (with a center wavelength of $633 \mathrm{~nm}$ ) have a clearly visual effect. The main reason is that a highresolution grayscale image can be realized by using any nanostructures with anisotropy (shown in Eq. S4). When the transmission axis of analyzer is rotated from 0 to $\pi / 4$, the grayscale image with watermark information is clear only when a red filter is inserted into an optical microscope. When we employ a broadband source (a quartz halogen lamp of the optical microscope) to illuminate the sample, the captured image has a low contrast. The reason is that the transmission-type nanobrick deviates from a perfect polarizer in a broadband range (shown in Fig. 6 of the main text). 


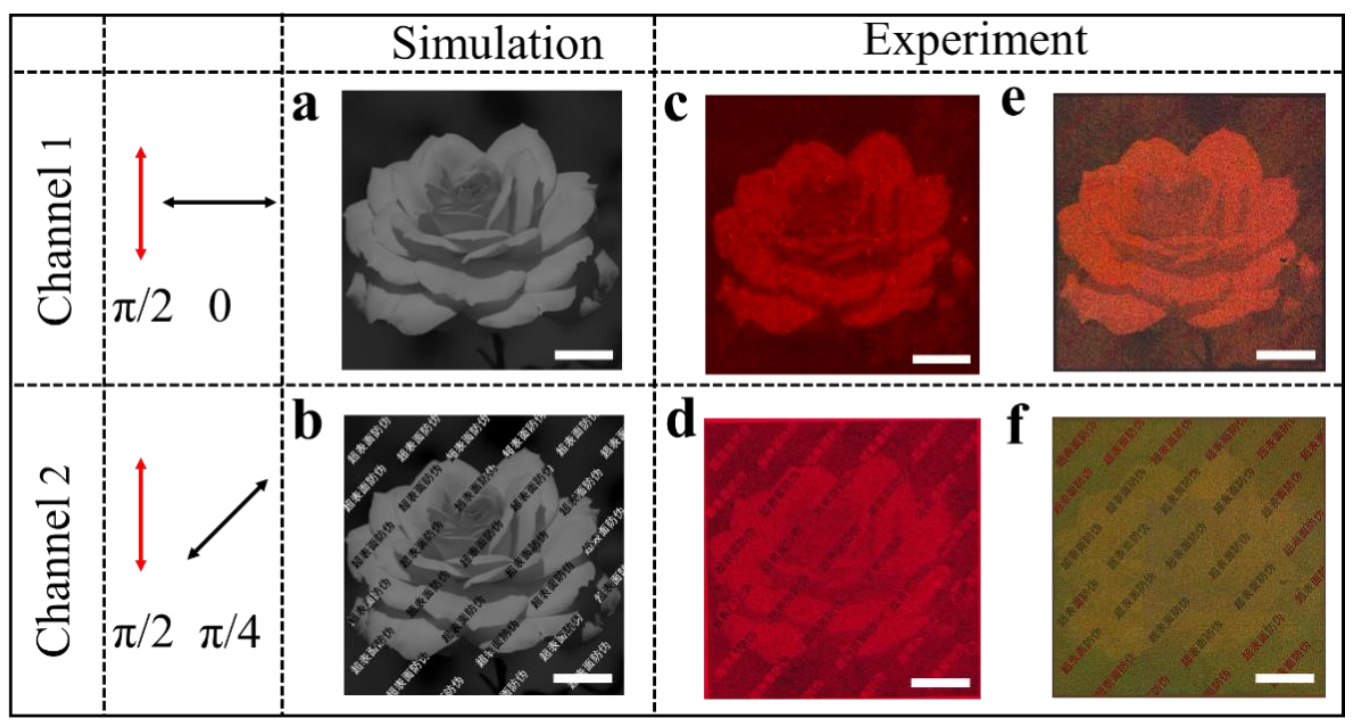

Figure S9. Simulation and experimental results of the sample four working in transmission. The first row is the meta-images of channel 1 when the transmission axes of the polarizer and analyzer are $\pi / 2$ and zero degree, respectively. The second row is the meta-images of channel 2 when the transmission axis of the analyzer is changed to $\pi / 4$. c, d, Captured images at an operating wavelength of $633 \mathrm{~nm}$ with the help of an optical microscope. $\mathbf{e}, \mathbf{f}$, Captured images under the illumination of an optical microscope's source. All samples are designed with dimensions of $200 \times 200 \mu \mathrm{m}^{2}$. The scale bars in all figures are $40 \mu \mathrm{m}$. The black and red arrows indicate the transmission axes of the polarizer and analyzer, respectively. 